\title{
Espace En Tant Qu'élément Essentiel Dans Un Théâtre
}

\author{
Busari Lasisi \\ Department Of French, Kaduna State University, Kaduna, Nigeria
}

\begin{abstract}
The playwright does not write his play in emptiness. His write up must be done in a given specific space bearing in mind that theatre is above all a spectacle. A theatre, being a short-lived performance, must by all means be presented before spectators who are watching. As a custom, one needs to study the function of space in a theatre, specifically that of Francophone Africa as well as her significance. That's what leads us to analyze the objectives of space in the play of Amadou Koné titled Le respect des morts (Respect for the dead)

Resume : Le dramaturge n'écrit pas sa pièce théâtrale dans le vide. Son écriture doit se situer dans un endroit spécifique quand il écrit sachant que le thêâtre est avant tout un spectacle. Le théâtre en tant qu'une performance éphémère doit coûte que coûte se produire devant des spectateurs qui regardent Il est de coutume d'étudier le rôle que joue l'espace dans une pièce théâtrale surtout celle de l'Afrique francophone voire sa signification. C'est ce qui nous amène à analyser les objectifs de l'espace dans la pièce théâtrale d'Amadou Koné intitulée Le respect des morts.
\end{abstract}

\section{Qu'est-ce que l'espace?}

\section{Introduction}

L'espace, selon Georges Peres, cité par Omonigho Stella, "est ce qui arrête le regard, ce sur quoi la vue bute. C'est-à-dire l'obstacle, des briques, un angle, un point de fuite" (109). Voyons aussi une autre définition donnée par Levy Leboyer cité toujours par Omonigho Stella selon laquelle " l'espace est le champ dans lequel se déploient la volonté et l'action humaine" (7). Des deux définitions, nous pouvons dire que l'espace est l'endroit où vivent les êtres humains (hommes et animaux). C'est aussi la place où les activités humaines de la vie se déroulent. Dans la littérature (le théâtre en particulier) l'espace est déterminé par le déplacement des personnages qui font les actions d'un endroit à l'autre. Dans une pièce théâtrale, l'espace peut être un lieu comme le village, le champ, le marché, l'église, la mosquée, la ville, le bureau, la buvette pour ne citer que ceux-ci. Il existe en littérature deux sortes d'espace (interne et externe), mais l'espace interne est ce qui nous concerne dans ce petit travail. L'espace interne est aussi appelé espace dramatique ou scénique, c'est-à-dire l'endroit où l'action se déroule dans une pièce de théâtre. Cet espace a pour rôle le pouvoir d'assister le lecteur à visualiser le moment où se déroule l'action. Il se trouve souvent dans le décor que le dramaturge présente à la tête de chaque acte.

\section{Presentation Bibliographique D'amadou Kone}

Amadou Koné est né en 1953 dans le petit village de Tanga dans le cercle de Banfana dans le Bukina Faso actuel. Il a eu sa formation scolaire dans son pays natal, la Côte d'Ivoire. Il est parti en France en 1977 où il a également eu son Doctorat de 3e cycle en littérature comparée à l'Université François-Diabelais (Tours). Pour ce qui est de sa carrière académique, de son retour de la France, il a enseigné la littérature africaine et comparée au Département de lettres modernes à l'Université d'Abidjan où il a eu l'occasion de préparer son Doctorat d'état es lettres qu'il a finalement obtenu en 1987 à l'Université de Limoges. Sa carrière a été bien exercée dans son pays natal aussi bien qu'ailleurs surtout aux Etats Unis. En 1992, il a été nommé maître assistant et puis maitre de conférence et gardera ce dernier jusqu'en 1997 quand il est devenu grand maître et membre du programme des cours africains à l'Université Georgetown. Son domaine étant la littérature africaine, il s'intéresse beaucoup aux genres littéraires oraux surtout dans le cadre de cinéma et l'histoire sociopolitique de l'Afrique subsaharienne. Comme chercheur et critique, il a étudié et publié des articles d'écrivains à un jeune âge. Déjà comme adolescent, il a commencé à produire des manuscrits et des recueils.

Amadou Koné était encore au lycée quand il a rédigé son premier roman qui ne serait publié que plus tard. Sa première nouvelle qui a vu le jour est Jusqu'au seuil de l'irréel publiée en 1976. Son premier roman d'écolier, une magnifique et tragique histoire d'auteur, Les frasques d'Ebinto est publié en 1980. Amadou Koné a publié au total six romans et plusieurs nouvelles et des pièces de théâtre dont certaines sont présentées dans le cadre du concours thêâtral interafricain de Radio France Internationale: Samory de Bissandougou en 1970, Sa sainteté le charlatan en 1971, De la chaire au trône en 1975, Le respect des morts en 1980, Les canaries sont vides en 1984. Il a aussi dirigé deux brillantes études sur la littérature orale africaine et le roman africain. Amadou Koné a encore à son actif la co-direction d'une Anthologie de la littérature de Côte d'Ivoire. 
L'oeuvre litteraire d'Amadou Koné a été honorée de nombreux prix. En 1981, Amadou Koné était membre de la fondation pro Helevetta de Zurich en Suisse. En 1985, il a reçu le prix du meilleur roman africain décerné par la Fondation Léopold Sédar Senghor. De 1990 à 1992, Amadou Koné est devenu membre de la prestigieuse Fondation Alexandre de Humbalt à Bonn en République Fédérale d'Allemagne.

Amadou Koné s'intéresse à la littérature orale africaine et aux nouveaux courants de la littérature moderne en Afrique. Les contes initiatiques et l'épopée aussi bien que leur influence sur le roman contemporain africain ont retenu toute son attention. Marié, Amadou Koné est père de deux filles et de deux garçons. Sa femme, Mary Lee Martin-Koné, est professeur d'Anglais à l'Université d'Abidjan.

\section{Compte Rendu De Le Respect Des Morts}

Le gouvernement, à travers le Commandant (le sous-préfet), annonce la construction d'un barrage afin de faire sortir un petit village de son état de sous-développement. Mais le village préfère attendre l'arrivée de Ndouba, le fils d'Anougba, le chef du village pour qu'il l'empêche. Son arrivée, si attendu, n'a pas résolu le problème car à l'annonce par le sous-préfet que les villageois doivent quitter leur propre village pour un autre, il y a ce refus véhément pour raison qu'ils ne veulent pas du tout quitter leur terre ancestral.

Afin de s'y opposer carrément, ils consultent les génies; une consultation qui comporte le sorcier du village, Anougba et le père de Ndouba également le chef du village. Ceux-ci révèlent l'intention des génies à tout le village: le sacrifice d'un nouveau-né. Cette demande troublante a nécessité une réunion. Après une décision prise, on convainc Ndouba de répondre positivement à la demande des génies puisqu'il est pour la construction du barrage. Ndouba accepte d'une manière difficile de sacrifier son fils Enokou. Ce sacrifice n'empêche pas la construction du barrage. Voici que les vieux s'apprêtent pour la lutte avec les prôneurs de la modernité.

\section{Espace: Element Essentiel Dans Un Theatre}

Nous allons essayer de diviser cet espace entre espace rural et espace urbain.

\section{Espace Rural}

L'espace rural est le type d'espace qui a affaire avec la campagne ou le village. Cela englobe les lieux comme la buvette, le champ, la rivière la chasse, etc... L'espace rural se rattache aux drames historiques et les légendes. Ici, on ne trouve que des choses primitives, démodées qui n'ont rien à faire avec la modernité. Des milieux où il n'y a ni de lumière, ni des routes goudronnées et des eaux potables. A titre d'exemple, dans Le respect des morts d'Amadou Koné que nous étudions, voyons le décor du premier acte:

Sous un petit hangar, quelques personnes boivent dans le pénombre. Dans un coin un peu plus éclairé, le Récitant commence son récit. (Koné, 9)

Le lecteur sait immédiatement qu'on ne peut pas avoir un hangar dans la ville, de même, cette sorte de buvette se trouve toujours dans les campagnes. Dans ce décor, on constate que ce hangar n'a pas d'électricité, tout est sombre, sauf un côté un peu éclairé, peut-être à cause de la lampe à huile allumée. Cela montre au lecteur le moment de la journée où on est. Pour aller plus loin, dans la première scène du quatrième acte dans Les derniers jours de Lat Dior d'Amadou Cissé, on assiste aussi à une description de l'espace rural ainsi:

A Touba. Un petit village perdu dans la brousse. Les cases apparemment misérables! C'est la nuit. Une lune ronde éclairée. Des bruits mystérieux: chants d'oiseaux, cris d'animaux sauvages! De partout, s'allume des feux de bois autour desquels .... (Cissé, 35)

Dans cette scène, nous n'avons rien vu de modernité. Toutes les actions et les objets mentionnés sont totalement traditionnels. Cette scène assiste le lecteur à avoir dans sa mémoire que les habitants de ce village sont loin de la vie moderne. Ils ne sont pas civilisés. Le lecteur n'a pas de problème de savoir le moment où se déroule la scène. Les gens ici vivent avec les animaux sauvages, ils mènent une vie primitive, raison pour laquelle ils s'assemblent pendant la nuit autour du feu de bois où ils content. Ils se servent des fagots de bois pour préparer leur nourriture, car il y manqué de l'électricité qu'on trouve toujours dans les villes.

La pièce de Tunde Ajiboye intitulée Olorunbi ou le prix d'un pari donne également un exemple clair d'un espace rural. Les actions et les rôles assignés à chaque personnage n'ont rien à faire avec la civilisation. Dans cette pièce, les personnages comme le chasseur, l'oracle, la vendeuse, le crieur du roi font partie des caractéristiques d'un espace rural. Voyons le décor au commencement de la pièce :

Les commerçants viennent un à un sur la scène pour étaler les articles dans leurs coins habituels au marché. Ils proclament hautement et, comme en chantant, la qualité de leurs étalages, en langue locale. Se présentent sur la scène des suivants; parmi d'autres, les passants. Les passants passent presque sans faire attention (Ajiboye, 10)

Dans ce marché qui sert comme espace dans cette scène, la langue locale est utilisée comme moyen de communication. Une autre personne qui vient de la ville (espace urbain) pour faire des achats doit coûte que coûte avoir besoin d'un interprète, qui est même l'un des personnages de la dite pièce. 
L'espace rural est aussi souvent déterminé par les noms que portent les divers personnages. Ici, il est difficile de trouver des acteurs qui portent des noms occidents. C'est la raison pour laquelle dans Olorunbi ou le prix d'un pari de Tunde Ajiboye, le lecteur trouve des acteurs comme Iya Ibeji, Maloma, Kabiyesi, Ogunranti, Awodina et ainsi de suite. L plupart des acteurs dans cette pièce portent des noms traditionnels, montrant ainsi le milieu où l'action a eu lieu. La maison du féticheur, c'est-à-dite chez l'oracle est également un exemple d'espace rural. Dans cet espace, on fait des sacrifices aux féticheurs. Il y a la récitation des incantations effrayantes. On y trouve également des choses primitives ou traditionnelles. Le féticheur se sert des proverbes pour véhiculer son message. Tunde Ajiboye est de cet avis lorsqu'il nous présente l'oracle dans la scène trois de l'acte trois de sa pièces en particulier

L'oracle : Tu vas vendre. La vipère "oka" ne bouge pas, néanmoins, elle trouve toujours à manger ; le python ne bouge pas, néanmoins, il trouve toujours à manger. "Montrez-moi des bagages, descendez-moi des bagages", c'est ainsi que parlent les termites, toujours ; le va-et-le vient est chose courante chez les fourmis. Le va-et-le vient des clients ne sera pas chose rare chez toi... (42-43)

Chez l'oracle, les joueurs de tam-tam sont toujours présents pour chanter l'éloge des visiteurs. Certains oracles ont besoin des interprètes pour faire passer leur message à leurs clients car ils utilisent des langues extraordinaires. Ces interprètes utilisent des gestes et des mimes (59)

La forêt en tant qu'espace rural est rarement utilisée dans les pièces francophones car il est difficile de le mettre en scène. Souvent, la forêt est présentée comme un espace remémoré. Dans l'espace rural, il y a un lieu où les gens se rencontrent pour boire des boissons locales. C'est ce qu'on appelle la buvette. Ici, les boissons locales comme ogogoro, le vin de palme sont vendues ou achetées. Toutes sortes de discussions sont faites dans la buvette. Les villageois arrivent des fois à se quereller s'ils ne s'attendent pas sur un sujet en discussion. Ils s'asseyent sur des tabourets par terre et se servent des calebasses pour boire le vin.

Dans l'acte premier de Le respect des morts d'Amadou Koné, le Récitant nous informe que les buveurs se servent du 'cango" pour boire le "'bangui" (9). La buvette se trouve sous un hangar couvert de feuilles des arbres. Les gens s'y rassemblent souvent le soir pour se divertir après les travaux durs de la journée.

L'espace rural est aussi représenté par la rivière qui traverse la plupart des campagnes africaines, d'ailleurs, c'est la raison pour laquelle bon nombre des pièces théâtrales des dramaturges africains sont liées à cet espace. Les habitants se nourrissent de l'eau de la rivière, de même, les oracles vont à la rivière consulter les génies qui s'y trouvent. Dans Le respect des morts d'Amadou Koné, ce sont les génies des eaux qui ont révélé aux villageois le sacrifice nécessaires pour sortir du danger qui les attend. Ecoutons cette scène :

Les génies des eaux demandent qu'un tout jeune enfant leur soit offert. Alors, ils empêchent la construction du barrage. Et les morts seront contents et nous regagneront la paix. Et nous garderons la paix. Voilà le remède, N'douba. Nous devons donner un enfant aux génies des eaux..., pour que notre village ne soit plongé à jamais dans le monde du silence (44-45)

Les femmes doivent aller à la rivière chercher de l'eau pour en servir dans les travaux domestiques. Elles y vont également pour laver leurs habits. Les hommes, de leur part y amènent leur troupeau pour boire de l'eau. Ils se servent également de l'eau de la rivière pour construire leur maison en banco. C'est au bord de la rivière que les sacrifices aux génies des eaux se font. La scène suivante dans Le zulu de Tchicaya U Tam'si atteste ce que nous disons : «Bruits de la nuit. On est au bord d'une rivière. Chants de crapaud. Tout est plongé dans la pénombre » (111) C'est là où la chute de Chaka a été organisée et exécutée par les duos de Ndlebe et Namdi. Les sorciers préfèrent entreprendre leurs actes cruels au bord de la rivière à cause de la solitude et du calme qui s'y est.

L'homme fort de la maison du féticheur, c'est-à-dire le sorcier est toujours un personnage terrible à cause de son habillement. Ce n'est pas tout le monde qui a accès à cet espace. Seuls les vieux peuvent y entrer. Les enfants et les jeunes femmes ont souvent peur de rentrer dans ce domicile du féticheur. Le décor de l'acte quatre de Trois prétendants... un mari de Guillaume Oyono- Mbia donne une description complète du sorcier dans son domaine :

Il fait déjà nuit. La chaine est éclairée par un grand feu autour duquel tous les villageois sont installés en demi-cercle. Sanga-Titi, le sorcier est un personnage à l'aspect terrible portant autour des reins des peaux de chats sauvages et de singes à longs poils. Son torse nu est badigeonné de kaolin. Il a sur la tête une coiffure faite de longues plumes de coqs et de toucan et quand il marche, les clochettes qu'il porte aux pieds résonnent joyeusement..... Encore une fois, il s'agit ici d'une intermède dansant. (Oyono-Mbia, 81)

Tout ce que le féticheur porte est effrayant. Cet espace a des odeurs désagréables à cause des sacrifices qu'on y fait tous les jours. Les féticheurs ont l'habitude de construire leur domaine près des rivières où ils peuvent se rendre facilement. Le domaine du féticheur est souvent appelé espace sacré car selon Echenim cité par Omonigho Stella «le féticheur est un nom générique représentant le guérisseur, le marabout-sorcier, le sorcier, le voyant, le sérigne » (14). Echenim est toujours de l'avis que :

Le féticheur traîne derrière lui une atmosphère qui lui donne une dimension extraordinaire sur le plan de l'acte accompli. Les attributs du féticheur le situent au delà du commun des mortels (14) 
Le décor de l'acte trois de Le respect des morts d'Amadou Koné nous vient rapidement à l'esprit comme un exemple clair d'un espace sacré :

Niangho est assez bizarrement accoutré : un petit pagne blanc autour des reins; le corps peint de kaolin; des files de cours entrecroisées sur la poitrine, d'autres attachées à ses poignets et à ses chevilles (43)

\section{Espace Urbain}

Cet espace a souvent eu lieu dans les villes et est des fois accompagné de comédies. Cet espace reflète la modernité connue à travers les personnages en scène, le décor et les objets utilisés. Parmi les différents objets qui se trouvent dans cet espace, nous pouvons citer : le bureau, le salon, le fauteuil, l'ordinateur ou la machine à taper, l'homme habillé à la française, la télévision, la radio, de l'électricité pour ne citer que ceux-ci.

Une pièce de théâtre une fois représentée par les objets énumérés tout à l'heure donne naissance à la civilisation et au modernisme. Considérons par exemple le décor suivant tiré du deuxième acte de Trois prétendants .... un mari de Guillaume Oyono- Mbia :

Même après-midi à M. Mvoutessi. Mbia est assis bien en évidence dans un grand fauteuil placé au milieu de la scène. Il a l'air vraiment important : costume en tergal du bon faiseur, lunettes de soleil, etc. Une formidable collection de médailles lui orne la poitrine. Engulu, son chauffeur, ira de temps en temps pour lui offrir des cigarettes à la ronde et les villageois enchantés en prendront toujours plus d'une à la fois. Pour n'être que le domestique du grand homme. Engulu n'en regarde pas moins tous ces broussards d'un peu haut, en bon citadin. Point n'est besoin de préciser que les femmes n'assistent pas à cette palabre au sommet. (29)

L'habillement de Mbia montre qu'il n'est pas un villageois. Les objets comme le fauteuil, les lunettes et les médailles n'ont rien à faire avec l'espace rural. Voilà un espace qui montre le modernisme à travers les objets et les personnages qui s'y trouvent. Mbia est une haute personnalité très riche avec un chauffeur qui le conduit partout où il va. Le bureau aussi est un endroit qui a affaire avec l'espace urbain parce que les objets qu'on y trouve sont des choses de la modernité à savoir des classeurs, des ordinateurs, des dossiers, des horloges affichés au mur, des climatiseurs et des ventilateurs. Ce type d'espace est décrit par Jean Pliya, l'écrivain béninois dans sa pièce théâtrale intitulée La secrétaire particulière ainsi :

La scène vide représente une vaste pièce avec deux armoires contre le mur, trois tables dont l'une supporte une pile de dossier, des classeurs, et les deux autres, deux machines à écrire. A côté, trois chaises. (Pliya, 7)

Les objets énumérés ici seront très étranges à un villageois qui n'est jamais sorti de son milieu rural. Le lecteur de ce décor que nous venons d'énumérer sait immédiatement que la scène ne peut jamais avoir lieu dans un milieu rural compte tenu de la qualité des personnages et la disposition des objets. Nous savons que dans les campagnes, les maisons sont construites avec de la boue de terre souvent appelées maisons en banco et couvertes de la paille ou des feuilles des arbres. Mais l'espace urbain comprend souvent des maisons construites avec du ciment et peinte avec de belles couleurs. Ces maisons sont couvertes de tôles et bien meublées. Un exemple clair et concret de ce que nous venons de souligner est le décor de la scène suivante tirée de La parenthèse de sang de Sony Labou Tansi :

Grande villa bleue. Sur la terrasse, une table qui attend. Dans la cour, trois jeunes kambala qu'on entendrait pousser, sous les kambala, trois tombes dont une relativement récente ..... (Labou Tansi, 6)

De même, le décor suivant de Le respect des morts d'Amadou Koné ne saute pas aux yeux quant à ce qui concerne l'arrangement d'un espace urbain :

Intérieur du palais, salle du trône richement meublée, bien tapissée. Le prince marche lentement de long en large. Il a le visage soucieux. Tranquillement assise dans un fauteuil, une jeune fille. Elle a un visage calme. Il fait nuit, mais la salle est assez bien éclairée. (Koné, 99)

Les deux décors ci-dessus montrent aux lecteurs que la scène ne se passe pas dans une campagne. En mentionnant une grande villa bleue, une salle richement meublée et tapissée, ce qui vient directement dans la tête est qu'il s'agit d'un espace urbain. On remarque que malgré la nuit, la salle est assez bien éclairée à cause de la disponibilité de l'électricité. Ce qui n'est pas le cas dans un espace rural où on allume pendant la nuit des lampes à huile locales.

Quant à ce qui concerne la prison en tant que lieu de détention et de torture, on peut l'avoir dans un espace rural et dans un espace urbain. La différence en est au niveau des composants de cette prison. Dans le théâtre traditionnel, les prisonniers peuvent se coucher par terre ou sur des vieux lits. Dans Le destin glorieux du Marechal Nnikon Prince qu'on sort de Tchicaya U Tam'si, le décor de la première séquence du premier plan nous informe que la scène représente une cellule de prison où il y a des lits défoncés (95). Cela montre que c'est une vieille prison démodée.

En allant plus loin, Sony Labou Tansi dans Antoine m'a rendu son destin, nous montre un exemple de prison moderne selon la description du décor suivant :

A la prison de Bracara. L'intérieur est devenu un véritable palais tandis que l'entrée et l'extérieur restent couverts de graffitis de toutes sortes et de crases. On voit au milieu de la cour un immense lit sur lequel 
les prisonniers viennent jeter, ainsi que des ordures, les tenues de ville qu'ils refusent de mettre contre leur uniforme numéroté. C'est le grand matin. Partout, on lit comme des signaux de circulation, en lettres gigantesques: «ECHANGEZ VOTRE BONNE CONDUITE CONTRE LA LIBERATION» «VIVE LA QUINZAINE DE SON ALTESSE ANTOINE ». «A BAS LES CROCODILES ET LES TORTURES » $\mathrm{Au}$ mât central pend une géante marionnette à trois couleurs que vent agite un peu. Un groupe de prisonniers assez je m'en foutistes chantent l'hymne des crocos (Labou Tansi, 24)

Ce décor montre que cette prison n'est pas un milieu traditionnel. Ici, les prisonniers sont éduqués puisqu'ils peuvent lire les instructions affichées au mur.

La rue est aussi un espace utilisé par les dramaturges. La rue peut être utilisée dans les deux espaces (rural et urbain). Elle peut signifier un espace glorieux et un espace fructrueux en fonction du décor. Dans les pièces jouées dans les campagnes, le griot ou le crieur du roi doit marcher tout au long de la rue avec son gong à la main pour convoquer les habitants au palais du roi ou pour leur passer des informations importantes de la part de ce dernier. Dans Olurounbi ou le prix d'un pari de Tunde Ajiboye, la scène deux qui a eu lieu sur la rue est un exemple clair :

CRIEUR DU ROI : (les sons du gong). C'est le roi qui me charge de vous adresser ces quelques mots. Ecoutez, écoutez la voix du roi. Grands et petits, approchez-vous! Hommes, femmes, approchez-vous! Approchez-vous pour bien entendre. To ! To ! Toto ! (les sons du gong) Au nom de Kabiyesi Onikoyi, j'ai â vous informer qu'après consultation avec le divinateur, le sort de notre pays serait entre les mains de nos ancêtres ...... mais soyons prêts à lui montrer notre reconnaissance enfin de compte (39-40) personnage.

Voilà une scène qui a eu lieu sur la rue dans un espace rural compte tenu du rôle joué par le

D'autre manière, quand nous considérons le premier acte de La fable du Cloitre des cimetières de Caye Makhele, la scène a eu lieu sur la rue d'un espace urbain :

Une rue Makiadi crache abondamment dans ses mains et se fait un brin de toilette, un chantonnant. Il se regarde avec plaisir dans un miroir brisé.... Il ouvre une vieille valise et en sort un vieux sur lequel sont accrochés divers objets - bouteille d'eau minérale, réveil, poste de radio, capsules, préservatifs, qu'il endosse. Il chante et danse ( Makhele, 19)

Les objets décrits ci-dessus montrent que la scène a eu lieu dans une rue de la ville. Ces objets montrent également les différentes activités qui se déroulent sur cette rue urbaine.

\section{Conclusion}

L'espace, en tant qu'élément essentiel dans un théâtre a été énuméré dans ce bref travail. Par le présent travail, on sait que le théâtre doit nécessairement se dérouler dans un espace. En tant qu'élément inévitable dans une pièce théâtrale, on ne peut pas parler de théâtre sans parler d'espace. Amadou Koné et ses collègues dramaturges africains n'utilisent pas l'espace dans leurs pièces pour ne rien dire. Chaque espace a un message essentiel à faire passer au public qui est le spectateur ou le lecteur. Comme nous l'avons distingué, il y a dans une pièce thêatrale l'existence d'un espace rural et urbain. Les objets qui s'y trouvent voire le comportement des personnages distinguent un espace de l'autre. Ceci dit, une pièce théâtrale doit coûte que coûte avoir un espace. L'espace a le rôle de divertir le public et aussi de le faire pleurer.

\section{References}

[1]. Omonigho, Stella. «Espace : forme et sens dans le théâtre francophone africain » KASUJOF :

[2]. Kaduna State University Journal of French 1.1 (2010): 181-201.

[3]. Koné, Amadou. Le respect des morts, Paris: Hatier, 1980.

[4]. Ajiboye, Tunde. Olurounbi ou le prix d'un pari, Ibadan : Bounty Press Limited, 2004.

[5]. Oyono-Mbia, Guillaume. Trois prétendants.... un mari, Yaoundé : Editions Clé, 2009.

[6]. Pliya, Jean. La secrétaire particulière, Yaoundé, CLE, 2001

[7]. U'Tamsi, Tchicaya. Le destin glorieux de Nnikon Nniku Prince qu'on sort, Paris : Présence

[8]. africaine, 1979.

[9].

[10]. Labou Tansi, Sony. Antoine m'a vendu son destin, Revue Equateur 1, 1986.

[11]. - --------- La parenthèse de sang, Paris : Hatier, 1979.

[12]. Makhele, Caye. La fable du cloître des cimetières, Paris : RFL, 1993. 